\title{
A Long-Duration Pre-flare Dip in the Light Curve of V1054 Oph
}

\author{
R. Ventura ${ }^{1}$, I. Pagano ${ }^{1}$, G. Peres ${ }^{2}$, M. Rodonó $\dot{o}^{1,3}$ \\ 1 Osservatorio Astrofisico di Catania, Città Universitaria, I-95125 Catania, Italy \\ 2 Istituto e Osservatorio Astronomico, Palazzo dei Normanni, I-90134 Palermo, Italy \\ 3 Istituto di Astronomia, Università degli Studi, viale A. Doria 6, I-95125 Catania, \\ Italy
}

Photometric observations of V1054 Oph (Wolf $630 \mathrm{AB}, \mathrm{m}_{V}=9.69$ ) and of the comparison star HD $152678\left(\mathrm{~m}_{V}=8.1\right)$ were carried out in the $U B V$ bands at Catania Astrophysical Observatory on 1994, June 15, using the $91 \mathrm{~cm}$ Cassegrain telescope equipped with an improved version of the twin-beam photometer URSULA (De Biase et al. 1988). We have detected a relatively low-intensity flare event preceded by a pre-flare dip of low amplitude and the exceptionally long duration of $36 \mathrm{~min}$. The integration times were 10,5 and $5 \mathrm{sec}$ in the $U, B$ and $V$ bands, respectively. The variable and the comparison stars were observed simultaneously in the two independent channels of the twin-beam photometer. The sky background was subtracted from the photon count rates of V1054 Oph and its comparison star in each spectral band, and the flux of the variable was normalized to that of the comparison star.

Owing to the very small angular separation $\left(0^{\prime \prime} \cdot 201\right)$ of the two brightest components of the system ( $\mathrm{A}$ and $\mathrm{B}$ components) the measured flux was that of the combined total flux of the system. Since both components are classified as flare stars, the observed activity phenomena may have occurred in any of the two stars. Therefore we have considered the possibilities that either A or B had flared. In both cases we removed the flux contribution of the non-flaring star from the observed combined flux.

Fig. 1 shows the differential light curves in the $U B V$ bands on 1994 June 15. The characteristics of the flare and the pre-flare dip are reported in Table 1.

Table 1. Magnitude variations at flare maximum and at dip minimum, and total energy in the three spectral bands involved in the two events. The corresponding flare values in the $V$ band is not reported because the $\Delta V$ was below our sensitivity level.

\begin{tabular}{|c|c|c|c|c|c|c|c|c|c|c|}
\hline & $\begin{array}{c}\Delta U \\
(\mathrm{mag})\end{array}$ & $\begin{array}{c}\Delta B \\
(\mathrm{mag}) \\
\mathbf{F L}\end{array}$ & $\begin{array}{c}E_{U} \\
(\text { erg }) \\
\mathbf{A R E}\end{array}$ & $\begin{array}{c}E_{B} \\
(\text { erg) }\end{array}$ & $\left(\begin{array}{c}\Delta U \\
(\mathrm{mag})\end{array}\right.$ & $\begin{array}{c}\Delta B \\
(\mathrm{mag})\end{array}$ & $\begin{array}{c}\Delta V \\
(\mathrm{mag})\end{array}$ & $\begin{array}{c}E_{U} \\
(\mathbf{e r g}) \\
\text { DIP }\end{array}$ & $\begin{array}{c}E_{B} \\
(\mathrm{erg})\end{array}$ & $\begin{array}{c}E_{V} \\
(\text { erg) }\end{array}$ \\
\hline $\mathbf{A}$ & 1.01 & 0.14 & \multirow[b]{2}{*}{$1.9310^{32}$} & \multirow[b]{2}{*}{$5.6810^{31}$} & 0.19 & 0.10 & 0.06 & \multirow[b]{2}{*}{$1.9710^{31}$} & \multirow[b]{2}{*}{$2.7510^{31}$} & \multirow[b]{2}{*}{$7.0810^{31}$} \\
\hline B & 1.14 & 0.17 & & & 0.23 & 0.12 & 0.07 & & & \\
\hline
\end{tabular}




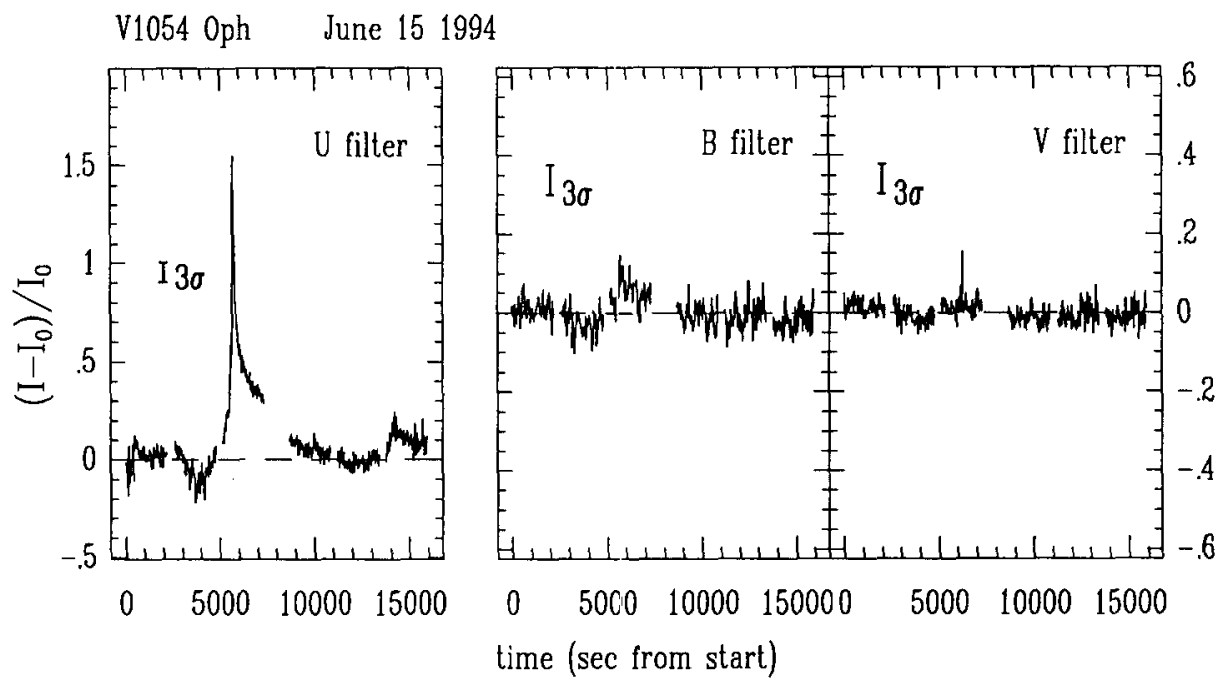

Fig. 1. The $U, B$ and $V$ light curves of the flare detected on V1054 Oph, on 1994 June 15 beginning at 22:59:54 UT. Time is reckoned in seconds from 21:00 UT. The ordinate axis scale is normalized to the quiescent star level, assuming that the flare had occurred on the A component. Note that the $B$ and $V$ plots are expanded by a factor of 2 with respect to the $U$ plot.

The present observation of such a long duration pre-flare dip is hardly reconcilable with any theoretical model of dips so far proposed (Gurzadyan 1968, 1980; Mullan 1975; Grinin 1976; Giampapa et al. 1982) and poses strong constraints on them.

\section{References}

De Biase G.A., Paternò L., Fedel B., Santagati G., Ventura R., 1988, 2nd Workshop on Improvements to Photometry, NASA CP-10015, Borucki (ed.), p. 17

Giampapa M., Africano J.L., Klimke A., Parks J., Quigley R.J., Robinson R.D., Worden S.P., 1982, ApJ 252, L39

Grinin V.P., 1976, Izv. Krymsk. Astrofiz. Obs. 55, 179

Gurzadyan G.A., 1968, Astrofiz. 4, 154

Gurzadyan G.A., 1980, Flare Stars, Pergamon, Oxford

Mullan D.J., 1975, A\&A 40, 41 